\title{
Experimental Measures of Bus Comfort Levels Using Kinematic Parameters Recorded by Smartphone
}

\author{
Salvatore Dell'Aquila, Laura Eboli, Giuseppe Futia, Gabriella Mazzulla, Giuseppe \\ Pungillo \\ University of Calabria, Italy
}

\section{SUMMARY}

Comfort on board plays an essential role in the levels of satisfaction of a bus service perceived by passengers. The aim of this paper is to propose a measure of comfort based on two kinds of data: perceptions of passengers (subjective data) and accelerations of bus (objective data). For the collection of subjective data a questionnaire was addressed to a sample of university students, while a smartphone, equipped with GPS device and 3-axis accelerometer, was used to record the accelerations. Based on the recorded parameters, we determined the thresholds of the acceleration values beyond which the level of comfort cannot be considered as good.

\section{INTRODUCTION}

Bus service quality depends on a series of different service aspects, which can be distinguished into characteristics that more properly describe the service (e.g. frequency of runs), and characteristics depending more on customer tastes and less easily measurable (e.g. comfort) (Eboli and Mazzulla, 2012). Just comfort on board can be surely considered as an important service aspect affecting bus service quality. So, to improve it can be a convenient strategy for attracting more customers.

Service attributes in general can be measured by a range of simple disaggregate performance measures which can be used for measuring the ability of a transit agency to offer services that meet customer expectations (Transportation Research Board, 1999). These measures can be considered as objective measures. Service quality levels can be also evaluated on the basis of transit user judgements. These judgements, which can be considered a subjective measure of service quality, generally derive from the well-known Customer Satisfaction Surveys (CSS), which help transit operators to identify which service quality factors are considered as the most important by their customers (Eboli and Mazzulla, 2009). Both these different kinds of transit performance measures can support transit agencies for monitoring, evaluating, and implementing improvements in service (de Ona et al., 2014).

In the past, measuring transit performance was very difficult, and collecting the data to evaluate transit systems was very costly. Recently, as a result of the widespread implementation of Intelligent Transportation Systems (ITS) and Advanced Public Transit System technologies (APTS), data collection is no longer a limiting factor (El-Geneidy et al., 2007).

Comfort on board is among those aspects which are more difficult to measure because it is rather affected by the tastes of the passengers. Comfort measurement may be the most 
challenging topic to be measured, just because it depends on the human perception and it is affected by several factors. Förstberg (2000) specifies some types of human-vehicle interaction variables that influence the user's comfort: human factors (as age and gender), environmental factors (as temperature, and noise), spatial factors (as work-space, seat shape, etc.) and dynamic motion factors. However, also for this service characteristic, objective measures can be considered for measuring service quality levels.

Comfort is a function of many different service aspects, linked to comfort of seats, or crowding levels, or vibrations on board and so on. As an example, if we consider the comfort aspect linked to the vibration on board, braking and turns, some kinematic parameters, especially accelerations, can be considered as significantly affecting passenger comfort. In this case, an exhaustive and convenient analysis of comfort on board should be effected on the basis of the accelerations recorded along paths covered by buses, together with the perceptions directly stated by bus passengers. The aim of this paper is just to provide a measure of comfort linked to specific aspects such as route roughness, and driving behaviour in terms of slowing down, braking, steering, and accelerations, by considering two kinds of data: subjective data, collected through interviews addressed to bus passengers who can provide their perceptions about comfort on board; objective data, consisting in kinematic parameters of the vehicle, and specifically accelerations. For the collection of subjective data, a questionnaire was appropriately designed and addressed to a sample of university students; while to record the accelerations a smartphone, equipped with GPS device and 3axis accelerometer, was used. A specific app allowed the acceleration and speed values to be recorded. The experimental study involves two lines that connect the city of Cosenza with the University of Calabria, sited in the southern Italy.

Based on the recorded parameters, we determined the thresholds of the acceleration values beyond which the level of comfort cannot be considered as good. Moreover, as the recorded data are instantaneous and geo-referenced, we can know at what points of the track the quantities calculated are below or above the threshold value.

Few recent studies proposed measures of comfort linked to vibrations on board (e.g. Bodini et al., 2013; Sekulic et al., 2013; Prashanth et al., 2013); however, they didn't use the smartphone for measuring comfort as in our case. The works by Lin et al. (2010) and Lin and Chen (2011) propose a comfort measure by using GPS and G-sensor of modern smartphones, but differently from our work, the authors do not consider also the subjective data, that is the passengers' judgements about comfort.

The rest of the paper is structured as follows. Firstly, we briefly discuss about comfort on board as a service aspect affecting service quality, by providing some literature references. Then, we describe the collection of the data, in terms of subjective and objective measures. The fourth section is about the description of the proposed method for analysing comfort and the presentation and discussion of the results. Finally, we propose a brief conclusive discussion of the work.

\section{EXPERIMENTAL CONTEXT}

\subsection{Collection of subjective data}


Experimental context of our study is represented by the University of Calabria, which is the major centre of interest in the urban area of Cosenza, where about 120,000 inhabitants lived in 2013. More than 30,000 students attend the university, which is staffed by 2,000 people. A single transit agency manages the urban bus lines. These lines are prevalently dedicated to the students. The service is available from 7.30 to 00.30 . Service frequency is 1 run every 60 minutes; only during peak-hours frequency is 1 run every 30 minutes.

On a working day, about 8,000 students travel by urban bus. Our experimental survey was realized in November 2014, involving a sample of about 500 students who live in the urban area and habitually travel by bus. Therefore, the sampling rate is about $6 \%$.

Two bus lines were analysed. The first line (U6) predominantly covers urban roads, but the last part of the route is along fast-flowing roads. The covered length is 11.2 kilometres, with 7 bus stops prevalently placed in the first part of the route. The average travel time is around 30 minutes, with an operating speed of about 26 kilometres per hours. The second line (U4) covers exclusively urban roads for a length of 10.7 kilometres; the number of bus stops is 14. The average travel time is around 35 minutes, with an operating speed of about 18 kilometres per hours; the wide difference between the two values of operating speed is obviously due to the different number of bus stops of the two lines.

The questionnaire was handed out on board by using self-administered survey technique. Practically, interviewers explained to groups of users the questionnaire, and users themselves compiled the questionnaire in order to make more interviews in the time of the run, which was not very long. 260 questionnaires were given on the buses of the first line, but only 142 returned fully completed; also for the second line a significant number of questionnaires was excluded from the analysis because of their incompleteness: 261 handed out questionnaires, and only 137 fully completed. This not very high response rates are obviously due to the method of administration of the interviews.

The questionnaire aimed to collect general information of the students and their perceptions on comfort levels. Specifically, four comfort aspects were evaluated: (1) comfort linked to level of crowding, seats availability and convenience; (2) noisiness and vibrations; (3) route roughness; (4) driving behaviour (due to slowing down, braking, steering, accelerations). For each question, users could choice among five levels of judgements, from 1 (low level of comfort) to 5 (high level of comfort).

The interviewed students are equally spread between male and female; as expected, the major part of them are aged between 22 and 25 years (57\%). The selected runs were between 10:00 a.m. and 6:00 p.m.; they were characterized by a not high level of overcrowding, in fact $83 \%$ of the students occupied a seat on the bus. Finally, the major part of the interviewees are habitual transit users, because $50 \%$ of them runs on the bus from 3 to 5 times in a week, and $23 \%$ more than 5 days in a week.

In the following, we report the percentages of response of the users about the perceived levels of comfort in terms of route roughness and driving behaviour. The frequency distributions of the users' judgements show that, in both cases, passengers are oriented towards bad judgements about comfort levels (figures 1). In fact, the judgements which correspond to a value of 1 or 2 summarized $83 \%$ for comfort linked to route roughness and 
$73 \%$ for comfort due to slowing down, braking, steering, accelerations (driving behaviour).
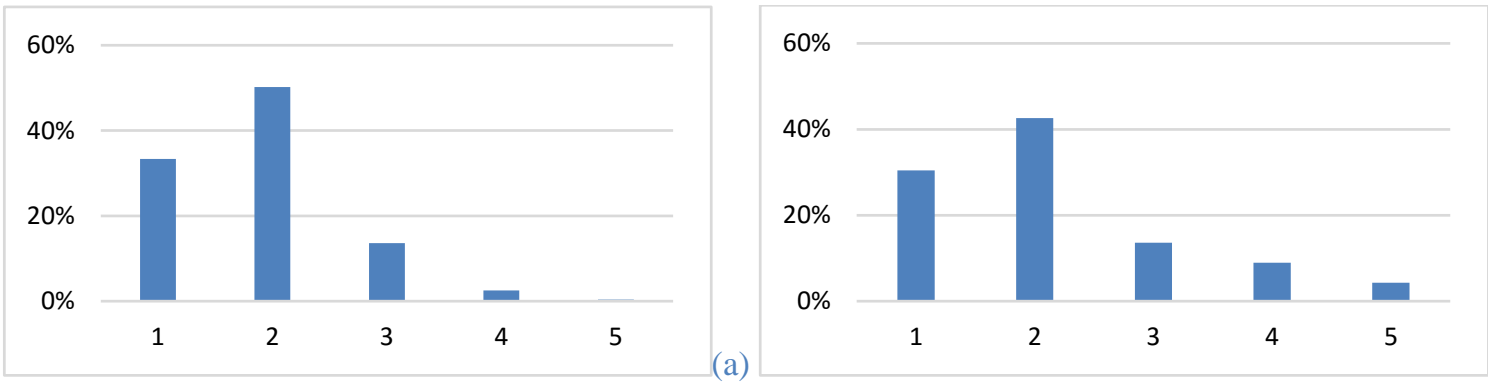

Fig. 1 - Frequency distribution of the judgements expressed about route roughness (a) and driving behaviour (b)

Considering these findings, we decided to focus our analysis on users' judgements expressed about only these two comfort aspects, in order to determine a threshold of some kinematic quantities beyond which you can objectively have a low level of comfort.

\subsection{Collection of objective data}

As above mentioned, objective data were collected by means of a smartphone equipped with GPS device and 3-axis accelerometer. A specific app (Torque) allowed the acceleration and speed values to be recorded, by adopting a frequency of 1 hertz, together with the instantaneous bus position (latitude and longitude), along the trajectory followed during the trip. Specifically, we recorded $G$ values in $x$-axis (longitudinal acceleration), in y-axis (lateral acceleration) and in z-axis (vertical accelerations). In order to have reliable data, smartphone was positioned on a horizontal plane, and a calibration of the system of reference with respect to the support plane was effected.

30 surveys were effected on Line U6, but only 19 paths were finally analysed because no GPS signal was captured by smartphone or the signal was distorted in a considerable part of the trajectory followed during the ride. For each survey an average value of 1,200 points was recorded. Analogously, 29 surveys were effected on Line U4, but only 17 paths were finally analysed. For each survey an average value of 1,700 points was recorded. All the surveys were graphically represented by using QGis.

\section{COMFORT LEVEL EVALUATION}

\subsection{Preliminary remarks}

We introduce a methodology for defining the level of bus comfort by using subjective and objective data. The methodology compares the level of comfort perceived on board by the passengers with the instantaneous values of the accelerations recorded by smartphone. Specifically, as we above stated, in this work we limit the analysis to the judgements expressed about two specific comfort aspects: comfort relating to driving behaviour due to slowing down, braking, steering, and accelerations, and comfort linked to route roughness. These judgements are then compared with $2 \mathrm{D}$ and $3 \mathrm{D}$ acceleration values, respectively. 
More specifically, we calculate the instantaneous 2D acceleration values by combining longitudinal and lateral acceleration values; in a similar manner, we calculate the instantaneous 3D acceleration values by introducing also vertical acceleration values.

A first analysis of the data consists in graphically representing the diagram of the $2 \mathrm{D}$ and $3 \mathrm{D}$ instantaneous acceleration values by highlighting in the graphs the thresholds of the positive and negative acceleration values. These thresholds were established by adding, for each survey, the average value of the accelerations and their standard deviation. We reproduced the diagrams for all the surveys, but we report in figure 2 the diagrams referred to a specific survey registered on Line U6.
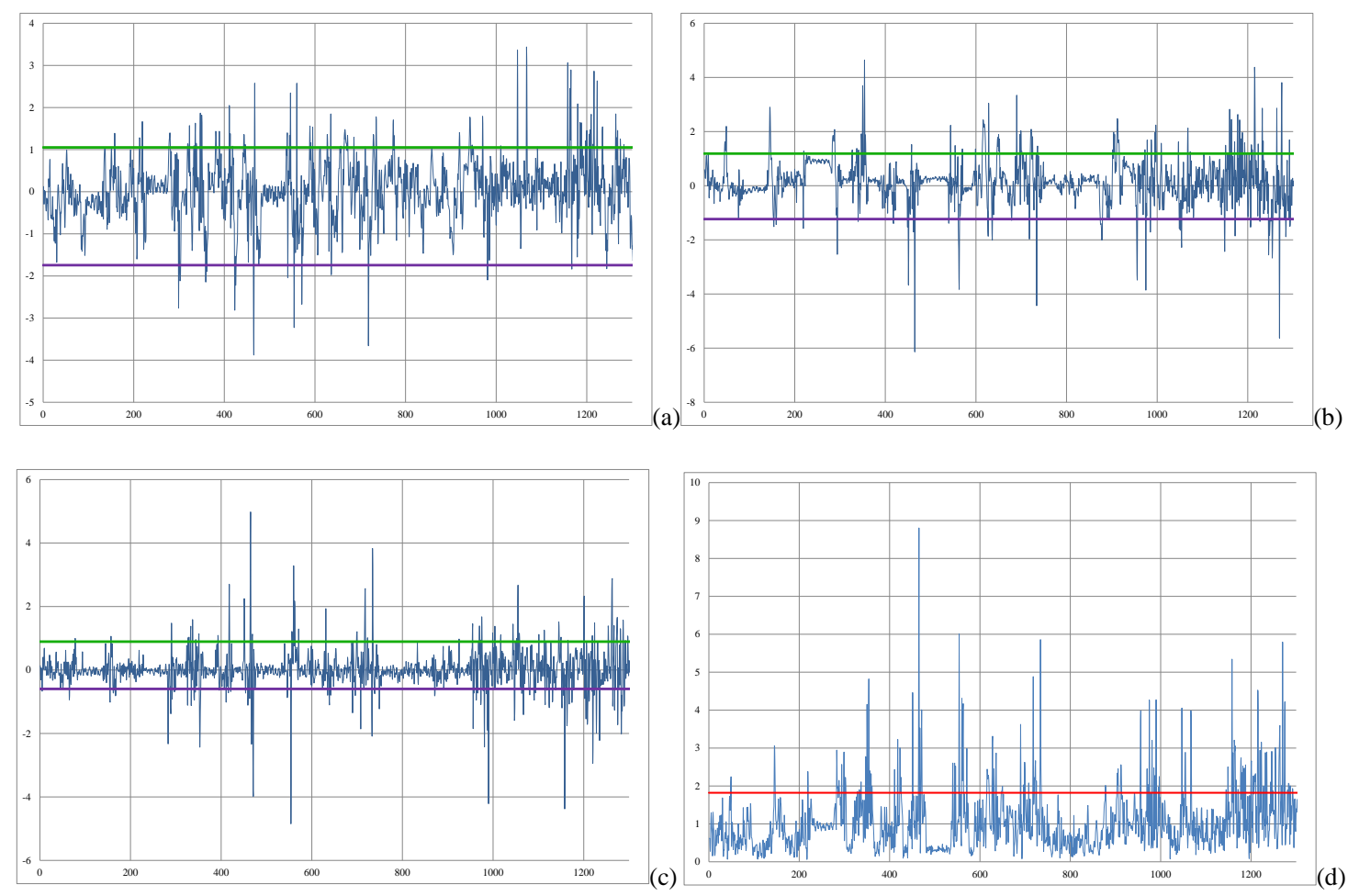

Fig. 2 - An example of graphical representation of longitudinal (a), lateral (b), vertical (c), 3D (d) acceleration values recorded on Line U6

From the figures, we can observe the values of the accelerations established as thresholds beyond which we can state that the level of comfort is objectively low. As an example, the thresholds relating to the longitudinal acceleration are about $1 \mathrm{~m} / \mathrm{s}^{2}$ and almost $-2 \mathrm{~m} / \mathrm{s}^{2}$, while the thresholds relating to the lateral acceleration are about 1.3 and -1.3 . If we consider the vertical acceleration, we observe thresholds absolute values lower than $1 \mathrm{~m} / \mathrm{s}^{2}$. Finally, the threshold for the $3 \mathrm{D}$ acceleration is almost 2. From the figures, we can also observe where the acceleration values go out of the established thresholds; in these cases, passengers stay in an uncomfortable situation.

A second step of the analysis consists in recording, for each survey, the number of points out of the thresholds, and calculating the rate of these outliers to the total recorded points. After this step, we can calculate the by the in order to establish a threshold value beyond 
which the level of comfort is low. More specifically, we obtained a series of rates by dividing the number of points out of the thresholds by the total recorded points. For each rate, we calculate the absolute frequency of the rate in the class representing the judgement expressed by the users; the class is defined by the judgements equal to 1 or 2 . We chose to make the analysis of the frequencies by considering only the judgements of those passengers who perceived a bad level of comfort, with the specific aim to determine a threshold of kinematic quantities beyond which the level of comfort is low, that is a situation of discomfort. We expect that the frequency distribution of the percentage values has an increasing trend, because the number of points out of the defined thresholds should increase in a situation of discomfort.

\subsection{Application}

By adopting the methodology above described, we analysed the surveys collected on the bus lines connecting the urban area of Cosenza with the University campus.

In Table 1 the number of points out of the thresholds for both 2D and 3D acceleration, and their rates to the total recorded points are reported for the survey of the Line U6.

\begin{tabular}{rrrrrr}
\hline \multirow{2}{*}{ Survey (N) } & \multirow{2}{*}{$\begin{array}{c}\text { Number of } \\
\text { points }\end{array}$} & $\begin{array}{c}\text { 2D acceleration } \\
\text { Number of points } \\
\text { out of the thresholds }\end{array}$ & Rate & $\begin{array}{c}\text { Number of points } \\
\text { out of the thresholds }\end{array}$ & Rate \\
\hline 1 & 1283 & 144 & 0,11 & 182 & 0,14 \\
2 & 1309 & 10 & 0,01 & 165 & 0,13 \\
3 & 1043 & 77 & 0,07 & 124 & 0,12 \\
4 & 1173 & 117 & 0,10 & 144 & 0,12 \\
5 & 1437 & 188 & 0,13 & 133 & 0,09 \\
6 & 1198 & 171 & 0,14 & 157 & 0,13 \\
7 & 1283 & 170 & 0,13 & 108 & 0,08 \\
8 & 1200 & 174 & 0,15 & 86 & 0,07 \\
9 & 1100 & 161 & 0,15 & 106 & 0,10 \\
10 & 1087 & 152 & 0,14 & 125 & 0,11 \\
11 & 1135 & 164 & 0,15 & 142 & 0,13 \\
12 & 1206 & 175 & 0,15 & 130 & 0,11 \\
13 & 1095 & 145 & 0,13 & 105 & 0,10 \\
14 & 1324 & 144 & 0,11 & 110 & 0,08 \\
15 & 1172 & 155 & 0,13 & 120 & 0,10 \\
16 & 1332 & 185 & 0,14 & 142 & 0,11 \\
17 & 1188 & 156 & 0,13 & 132 & 0,11 \\
18 & 1215 & 139 & 0,12 & 99 & 0,08 \\
19 & 1058 & 140 & 0,13 & 94 & 0,09 \\
\hline
\end{tabular}

Table 1 - Synthetic values (Line U6)

The rates reported in table 1 were used for calculating the absolute frequency values of the passengers' judgements. The frequency distributions are represented in Figure 3 and 4. By analysing the frequency distributions, we can observe that in the first case (figure 3) we have an increasing trend of the rate values, indicating that when the level of comfort is bad, the rate of the $2 \mathrm{D}$ acceleration values out of the thresholds increases. In the second case (figure 4), the shape of the frequency distribution is very similar to a normal curve. So, we decide 
to adopt as threshold values the modal value of the distributions.

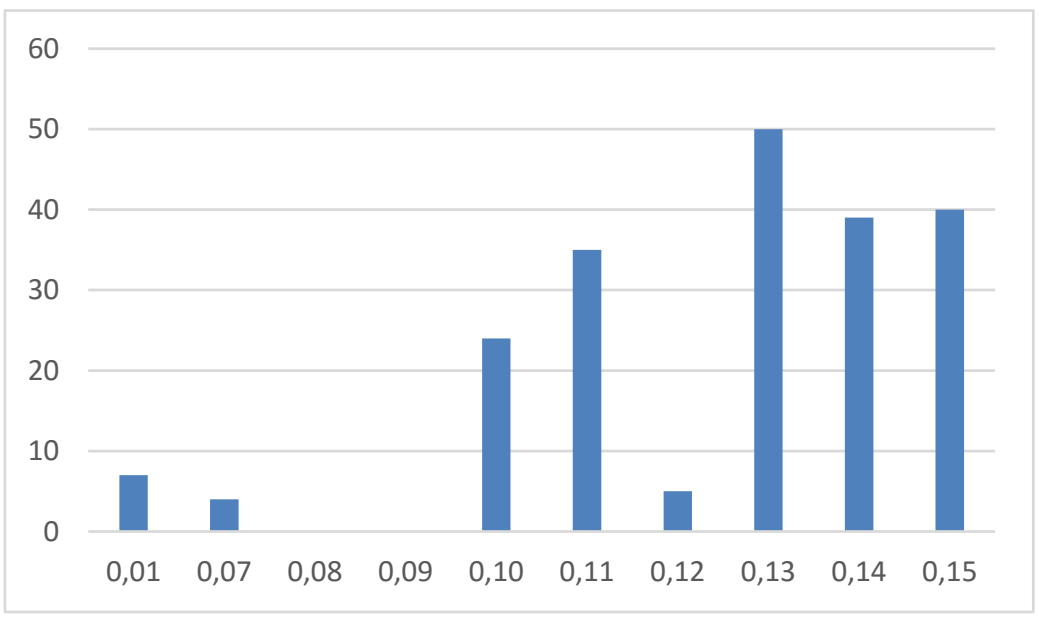

Fig. 3 - Frequency distribution of the level of comfort by using the percentage of the number of points out of the $2 \mathrm{D}$ acceleration values thresholds

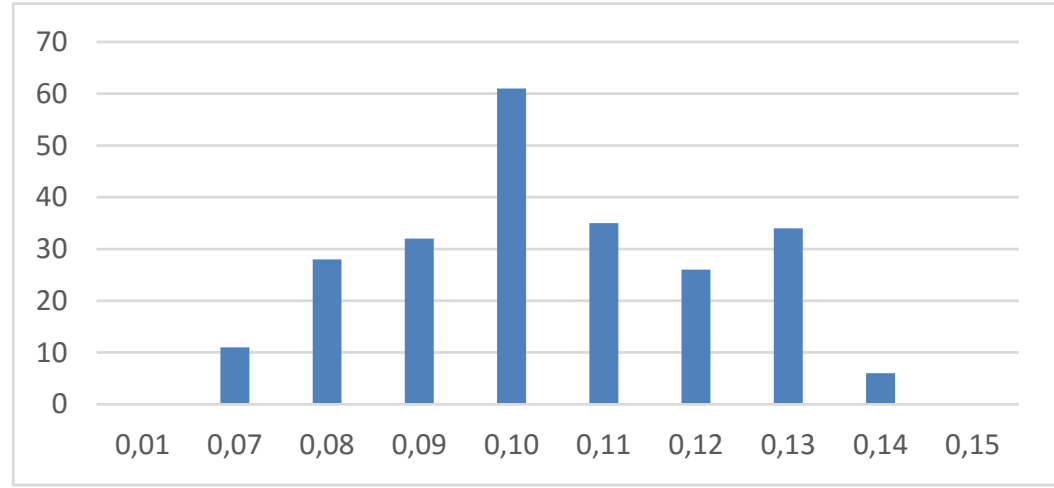

Fig. 4 - Frequency distribution of the level of comfort by using the percentage of the number of points out of the 3D acceleration values thresholds

On the basis of a preliminary analysis of the data, we can conclude that a threshold values of 0.13 could be adopted for indicating a low level of comfort on board linked to the driving behaviour (sudden acceleration and deceleration). More specifically, if for a certain bus ride the rate of the $2 \mathrm{D}$ acceleration instantaneous values is higher than 0.13 we can conclude that the level of comfort on board is bad; on the contrary, if the rate is lower than 0.13 the level of comfort is good. In the same manner, a threshold values of 0.10 could be adopted for indicating a low level of comfort on board linked to the route roughness.

\section{CONCLUSIONS}

In this paper, we focused on the analysis of a specific and very important bus service quality characteristic, that is comfort on board. We analysed particular aspects of comfort linked to the route roughness and to the slowing down, braking, steering, and accelerations that determine the behaviour of driving. We analysed comfort levels by considering both objective data obtained from the kinematic parameters registered thanks to the use of the 
smartphone, and subjective data representing by the judgements of the passengers about comfort perceived on board. The specific aim of this paper was to present a method for establishing thresholds of comfort level that can be adopted for defining if comfort is good or not. Through an analysis of frequency distribution we have reached interesting results and provide for a simple method for evaluating comfort levels by considering the subjective opinions of the passengers together with objective data based on the acceleration values, which give a correct measure of the comfort aspects.

\section{REFERENCES}

BODINI, I.; LANCINI, M.; PASINETTI, S.; VETTURI, D. (2013). Techniques for onboard vibrational passenger comfort monitoring in public transport. 12th IMEKO TC10 Workshop on Technical Diagnostics New Perspectives in Measurements, Tools and Techniques for Industrial Applications June 6-7 2013, Florence, Italy.

DE OÑA, R.; EBOLI, L.; MAZZULLA G.(2014). Key Factors Affecting Rail Service Quality in the Northern Italy. A Decision Tree Approach. Transport 29, pp 75-83.

EBOLI, L.; MAZZULLA G. (2009). An ordinal logistic regression model for analysing airport passenger satisfaction. Euromed Journal of Business 4(1), pp 40-57.

EBOLI, L.; MAZZULLA G. (2012). Performance indicators for an objective measure of public transport service quality. European Transport 51, pp 1-21.

FORSTBERG, J. (2000). Ride comfort and motion sickness in tilting trains: Human responses to motion environments in train experiment and simulator experiments. $P h D$ thesis, KTH Royal Institute of Technology.

LIN CHENG-YU; CHEN LING-JYH (2011). TPE-CMS: A Comfort Measuring System for Public Bus Service in Taipei City. The 30th IEEE International Conference on Computer Communications (Infocom'11), Shanghai, China, 2011.

LIN CHENG-YU; CHEN LING-JYH; CHEN YING-YU; AND LEE WANG-CHIEN (2010). A Comfort Measuring System for Public Transportation Systems Using Participatory Phone Sensing. International Workshop on Sensing for App Phones (PhoneSense'10, in conjunction with ACM SenSys'10), Zurich, Switzerland, 2010.

PRASHANTH, A.S.; SARAN, V. H.; HARSHA S.P. (2013). Study of subjective responses on ride comfort in public transport Uttarakhand State buses. 1st International \& 16th National Conference on Machines and Mechanisms (iNaCoMM 2013).

SEKUliĆ, D.; DEDOVIĆ, V.; RUSOV, S.; ŠAliniĆ, S.; OBRADOVIĆ, A. (2013). Analysis of vibration effects on the comfort of intercity bus users by oscillatory model with ten degrees of freedom, Applied Mathematical Modelling 37(18), pp.8629-8644.

TRANSPORTATION RESEARCH BOARD (1999). A handbook for measuring customer satisfaction and service quality, TRCP Report 47, National Academy Press, Washington, D.C. 